\title{
CMEARTICLE
}

\section{Clinics in diagnostic imaging (175)}

Vijay Krishnan ${ }^{1}$, MD, FRCR, Tze Chwan $\underline{\mathrm{Lim}}^{1}$, MBBS, FRCR, Francis Cho Hao $\underline{\mathrm{HO}}^{2}$, MBBS, FRANZCR, Wilfred CG $\underline{\mathrm{Peh}}^{1}$, FRCP, FRCR

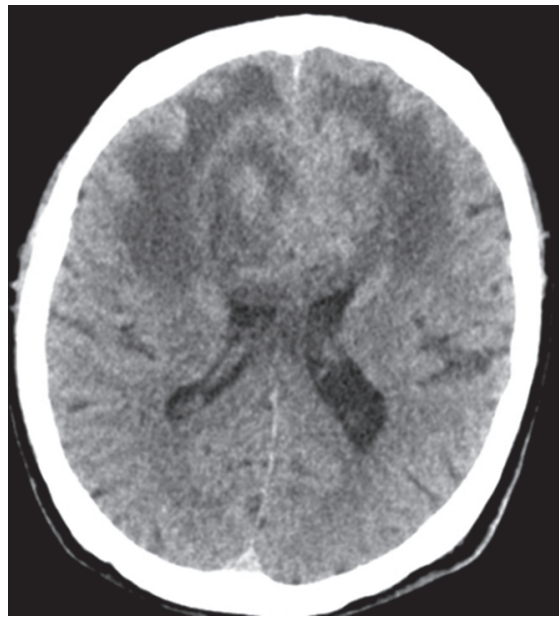

Fig. 1 Axial non-enhanced CT image of the brain
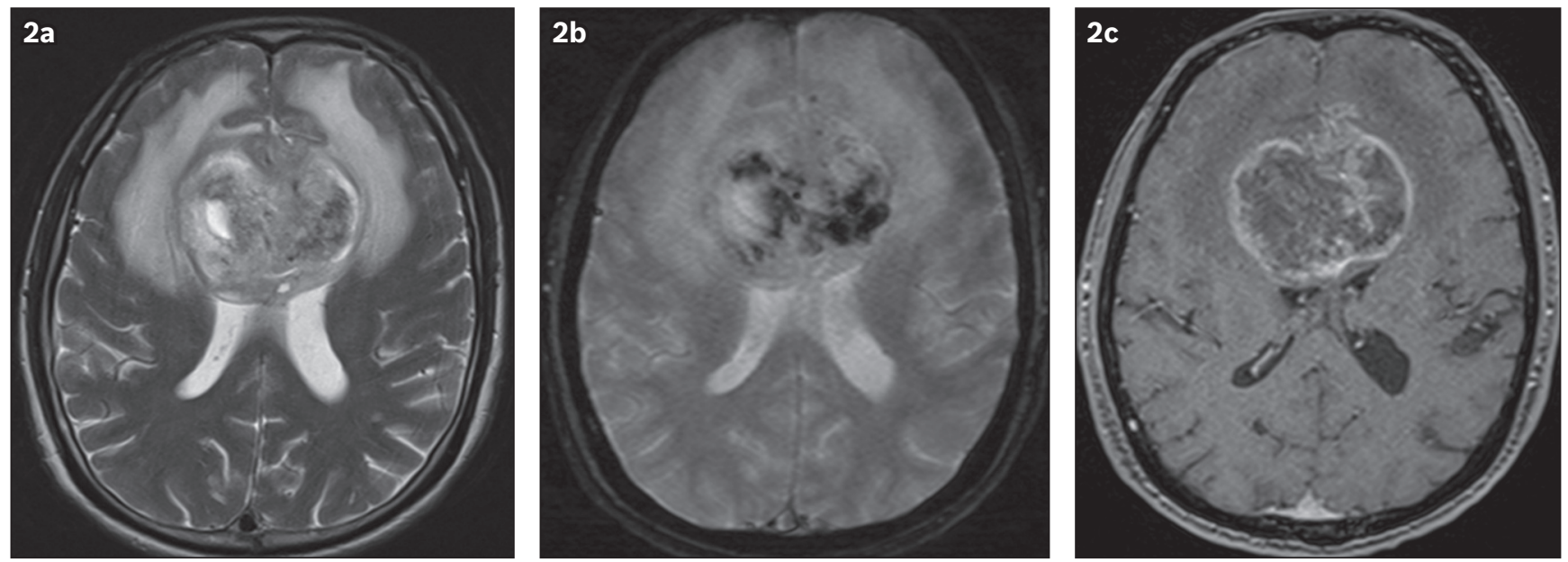

Fig. 2 Axial (a) T2-W, (b) gradient-echo and (c) enhanced T1-W MR images of the brain.

\section{CASE PRESENTATION}

A 54-year-old man presented with change in behaviour, nocturnal enuresis, abnormal limb movement and headache of one week's duration. His neurological examination was unremarkable. What do computed tomography (CT) (Fig. 1) and magnetic resonance (MR) imaging (Figs. 2a-C) of the brain show? What is the diagnosis? 


\section{IMAGE INTERPRETATION}

Unenhanced CT of the brain (Fig. 1) shows a heterogeneous lesion involving both frontal lobes, which crosses the midline at the genu of the corpus callosum. The lesion is associated with perilesional oedema and is causing a mass effect on the lateral ventricles anteriorly. MR imaging shows that the lesion has heterogeneous signal intensity with surrounding oedema and mass effect, best demonstrated in the T2-weighted sequence (Fig. 2a). The gradientecho image (Fig. 2b) shows multifocal areas of intralesional susceptibility indicative of internal haemorrhages. The contrastenhanced T1-weighted MR image (Fig. 2C) shows heterogeneous and predominantly peripheral enhancement of the lesion, with central non-enhancing areas indicative of intralesional necrosis. The lesion has a 'butterfly' configuration that is well depicted, especially on MR imaging.

\section{DIAGNOSIS}

Corpus callosum glioblastoma multiforme (GBM): butterfly glioma.

\section{CLINICAL COURSE}

Stereotactic biopsy of the tumour was performed; histopathology revealed pleomorphic atypical cells with hyperchromatic nuclei that were strongly positive for glial fibrillary acidic protein. The overall findings were consistent with glioblastoma multiforme. The tumour was considered inoperable due to associated postoperative morbidity, tumour histology, extensive size and the involvement of the corpus callosum. The patient was treated with dexamethasone to control the cerebral oedema and phenytoin to prevent seizure. He also underwent 12 fractions of palliative radiotherapy after being seen by a radiation and medical oncologist, and was then referred to hospice care for palliative management.

\section{DISCUSSION}

GBM is more commonly seen in patients older than 50 years of age. ${ }^{(1)}$ Imaging of these tumours usually shows a heterogeneous pattern of enhancement, with significant perilesional oedema and mass effect. Intralesional haemorrhage and necrosis may occur. They are usually solitary tumours. Restricted diffusion may be seen on MR imaging due to the high cellularity of these lesions. They also tend to show increased relative cerebral blood volume (rCBV) in perfusion imaging. ${ }^{(1,2)}$

Several neoplastic and non-neoplastic conditions may involve the corpus callosum and mimic a butterfly glioma. As the corpus callosum is usually resistant to infiltration by tumours, a mass that involves and crosses the corpus callosum is indicative of an aggressive neoplasm such as GBM. ${ }^{(2)}$ Lymphomas (Figs. 3a \& b) and brain metastases (Figs. $4 \mathrm{a} \&$ b) are other tumours that can infiltrate the corpus callosum, causing a similar 'butterfly' appearance. Non-neoplastic lesions such as tumefactive demyelination and toxoplasmosis can also involve and cross the corpus callosum, mimicking a butterfly glioma. ${ }^{(2,3)} \mathrm{A}$ meningioma, when it arises from the falx cerebri and crosses the midline, is another possible mimic (Figs. 5a \& b). ${ }^{(4)}$

Primary central nervous system lymphomas are usually of the B-cell non-Hodgkin type, which peak in incidence at the sixth decade of age. (1) Patients with congenital immunodeficiency and acquired immunodeficiency syndrome, as well as organ transplant recipients, are at high risk of developing lymphoma. In contrast to GBM, lymphomas tend to show less perilesional oedema and are less frequently necrotic. About half of these patients exhibit multiple lesions. (1) Lymphomas may show a hyperdense CT appearance and are usually homogeneously isointense to hypointense in T1-weighted MR images, as well as hypointense in T2-weighted MR images. They tend not to calcify nor bleed. Mild contrast enhancement and restricted diffusion may be seen in such lesions. In immunocompromised patients, a lymphoma may demonstrate heterogeneous signal intensity and ring enhancement. They are highly radiosensitive and may reduce or even vanish in response to steroid administration. $^{(1,2,5)}$

In multiple sclerosis (MS), multiple ovoid-shaped white matter plaques are seen perpendicular to the lateral ventricles.
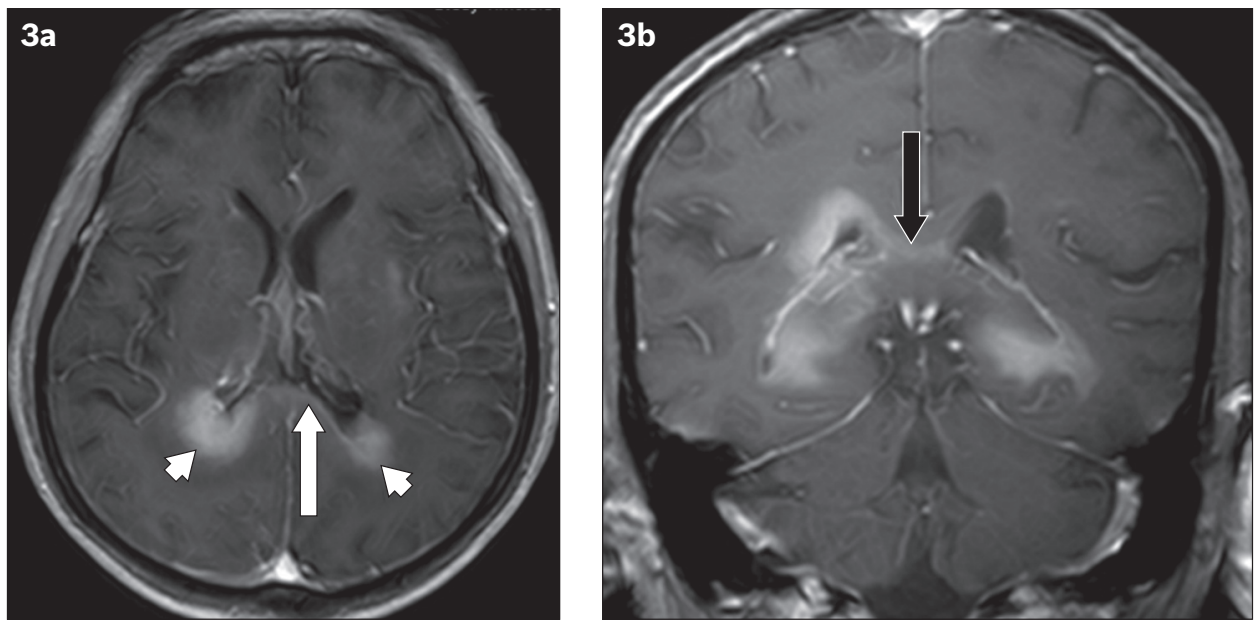

Fig. 3 A 69-year-old man with primary central nervous system B-cell lymphoma. (a) Axial enhanced T1-W MR image shows homogeneous enhancement of the lesion involving the periventricular region (arrowheads); there is also enhancement of the splenium of the corpus callosum (white arrow). The hypointense signal surrounding the lesion represents perilesional oedema, which is considerably less than that expected for a GBM or metastasis. (b) Coronal contrast-enhanced T1-W MR image shows abnormal enhancement of the corpus callosum (black arrow). 

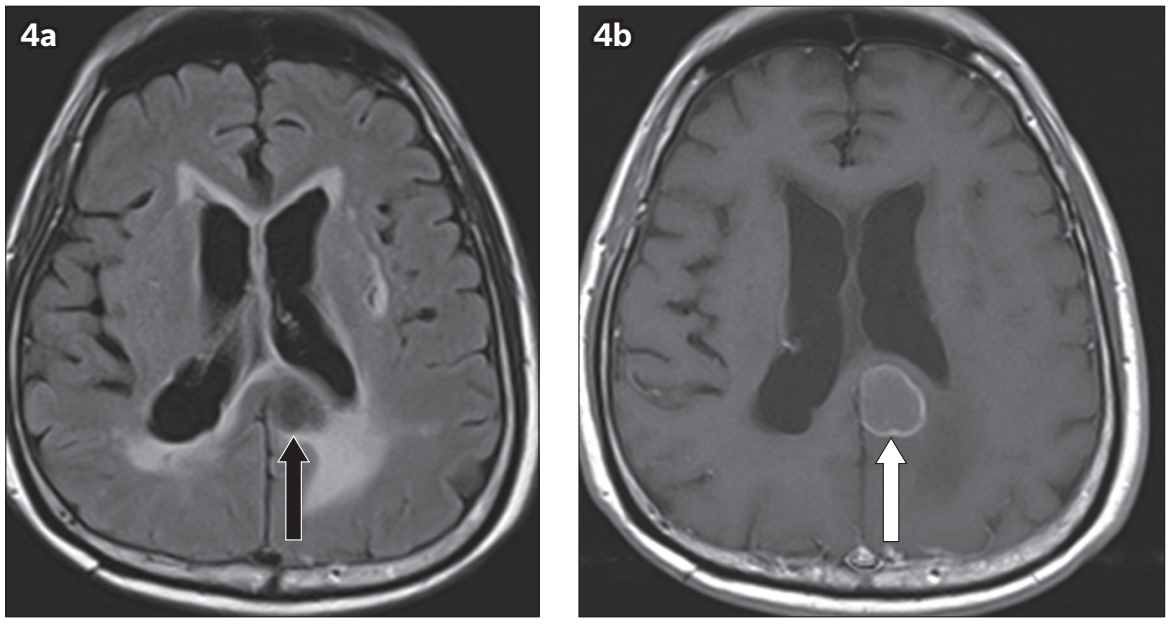

Fig. 4 Brain metastasis in a 72-year-old man with a history of known colonic malignancy. (a) Axial fluid-attenuated inversion recovery MR image shows a hypointense lesion involving the splenium of the corpus callosum (black arrow). (b) Axial enhanced T1-W MR image shows peripheral enhancement of the lesion (white arrow).
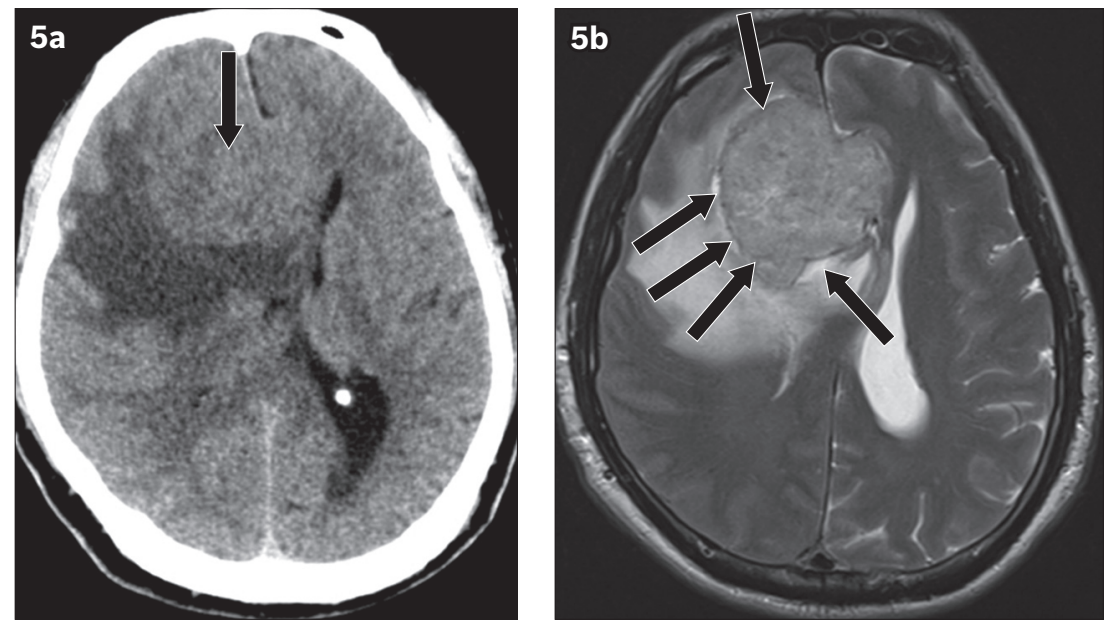

Fig. 5 Meningioma in a 58-year-old man presenting with right facial droop and left lower limb weakness. (a) Axial CT image shows an isodense lesion that is crossing the midline and closely related to the genu of the corpus callosum (arrow); it mimics a butterfly glioma. (b) Axial T2-W MR image shows a cerebrospinal fluid cleft (arrows) between the lesion and adjacent brain parenchyma, indicating the extra-axial location of the tumour.

Tumefactive demyelination is a type of MS that mimics a neoplasm in imaging. When it involves the corpus callosum, it may cross the midline and mimic a butterfly glioma (demyelinating butterfly pseudo-glioma). The presence of the following imaging features favours the diagnosis of MS over high-grade glioma: (a) an open ring-like enhancement; (b) minimal perilesional oedema in a large lesion; (c) dilated vein within the lesion, (d) decreased rCBV in perfusion imaging, and (e) resolution of the lesion after steroid therapy. ${ }^{(6)}$

Toxoplasmosis can affect patients with acquired immunodeficiency syndrome. Lesions usually present as multiple hypodensities with ring-like or nodular enhancement on CT, and are commonly located at the basal ganglia and corticomedullary junction. The enhancing lesion can mimic a tumour; when it involves the corpus callosum, crossing the midline, it can produce a 'butterfly' appearance. ${ }^{(7)}$

Metastasis is one of the differentials for lesions involving the corpus callosum. When the metastasis crosses the midline, it can mimic a butterfly glioma. ${ }^{(7)}$ The presence of multiple lesions and a known history of primary cancer are the key features in differentiating metastases from GBM. However, differentiating solitary metastases from GBM is challenging because of a similar imaging appearance on CT and conventional MR sequences. Tsougos et al described applications of advanced MR sequences such as MR spectroscopy and MR perfusion in differentiating these two lesions. ${ }^{\left({ }^{8}\right)}$ They described peritumoral ratio as the ratio of a particular parameter in the peritumoral area to that of the contralateral normal area. The authors concluded that elevated peritumoral ratio of rCBV in perfusion imaging, and elevated peritumoral ratios of $\mathrm{N}$-acetylaspartate (NAA)/creatine $(\mathrm{Cr})$, choline $(\mathrm{Cho}) / \mathrm{Cr}$, and Cho/NAA ratios in MR spectroscopy are useful imaging findings in differentiating GBM from metastases.

Meningiomas are extra-axial tumours often seen in middleaged female patients. They appear mildly hyperdense on unenhanced CT and show prominent homogeneous enhancement after administration of intravenous contrast. A meningioma can mimic butterfly glioma when it arises from the falx cerebri and crosses the midline. ${ }^{(3)}$ Presence of a cerebrospinal fluid intensity cleft between the tumour and adjacent brain cortex is a useful sign 
to identify the extra-axial location of these lesions and differentiate them from butterfly gliomas. ${ }^{(9)}$

In conclusion, GBM is the most common cause of callosal tumours with a 'butterfly' appearance. Other neoplastic causes include lymphoma, intracranial metastasis and falx meningioma. Non-neoplastic causes like tumefactive demyelination and toxoplasmosis can also mimic butterfly tumours of the corpus callosum. An adequate knowledge of imaging findings and

ABSTRACT A 54-year-old man presented with change in behaviour, nocturnal enuresis, abnormal limb movement and headache of one week's duration. The diagnosis of butterfly glioma (glioblastoma multiforme) was made based on imaging characteristics and was further confirmed by biopsy findings. As the corpus callosum is usually resistant to infiltration by tumours, a mass that involves and crosses the corpus callosum is suggestive of an aggressive neoplasm. Other neoplastic and non-neoplastic conditions that may involve the corpus callosum and mimic a butterfly glioma, as well as associated imaging features, are discussed.

Keywords: butterfly glioma, butterfly tumours, corpus callosum, glioblastoma multiforme prudent correlation with clinical findings will help in achieving early accurate diagnosis and appropriate patient management without unnecessary intervention.

\section{REFERENCES}

1. Jayaraman MV, Boxerman JL. Adult Brain Tumors. In: Atlas SW. Magnetic Resonance Imaging of the Brain and Spine, 4th ed. Philadelphia, PA: Lippincott Williams \& Wilkins, 2009: 446-590.

2. Bourekas EC, Varakis K, Bruns D, et al. Lesions of the corpus callosum: MR imaging and differential considerations in adults and children. AJR Am J Roentgenol 2002; 179:251-7.

3. Agrawal A. Butterfly glioma of the corpus callosum. J Cancer Res Ther 2009; 5:43-5.

4. Buetow MP, Buetow PC, Smirniotopoulos JG. Typical, atypical, and misleading features in meningioma. Radiographics 1991; 11:1087-106.

5. Krupa K, Bekiesinska-Figatowska M. Congenital and acquired abnormalities of the corpus callosum: a pictorial essay. Biomed Res Int 2013; 2013:265619.

6. Kazi AZ, Joshi PC, Kelkar AB, Mahajan MS, Ghawate AS. MRI evaluation of pathologies affecting the corpus callosum: A pictorial essay. Indian J Radiol Imaging 2013; 23:321-32.

7. Lee HJ, Williams R, Kalnin A, Wolansky L. Toxoplasmosis of the corpus callosum: another butterfly. AJR Am J Roentgenol 1996; 166:1280-1.

8. Tsougos I, Svolos P, Kousi E, et al. Differentiation of glioblastoma multiforme from metastatic brain tumor using proton magnetic resonance spectroscopy, diffusion and perfusion metrics at 3 T. Cancer Imaging 2012; 12:423-36.

9. Watts J, Box G, Galvin A, et al. Magnetic resonance imaging of meningiomas: a pictorial review. Insights Imaging 2014; 5:113-22. 


\section{SINGAPORE MEDICAL COUNCIL CATEGORY 3B CME PROGRAMME} (Code SMJ 201703A)

Question 1. Regarding corpus callosal tumours:

(a) The corpus callosum can be easily infiltrated by tumours.

(b) Glioblastoma multiforme (GBM) should be considered as the primary differential diagnosis for lesions involving the corpus callosum that have a 'butterfly' pattern.

(c) Non-neoplastic lesions such as toxoplasmosis can involve the corpus callosum and mimic butterfly gliomas.

(d) A meningioma, when arising from the falx cerebri, can mimic a butterfly glioma.

Question 2. Regarding GBM:

(a) GBM is more commonly seen in adolescents.

(b) GBM usually shows a homogeneous pattern of enhancement, without significant perilesional oedema.

(c) Intralesional haemorrhage and necrosis may occur.

(d) GBM tends to show increased relative cerebral blood volume (rCBV) in perfusion imaging.

Question 3. Regarding primary central nervous system lymphomas:

(a) They are usually of the B-cell non-Hodgkin type.

(b) They are more commonly seen in adolescents.

(c) Patients with congenital immunodeficiency and acquired immunodeficiency syndrome are at risk of developing primary central nervous system lymphoma.

(d) In contrast to GBM, lymphomas tend to show more perilesional oedema and are more frequently necrotic.

Question 4. In differentiation of lesions involving the corpus callosum, the following imaging characteristics favour the diagnosis of tumefactive demyelination over GBM:
(a) An open ring-like enhancement.
(b) Gross perilesional oedema in a small lesion.
(c) Increased rCBV in perfusion imaging.
(d) Resolution of the lesion after steroid therapy.

Question 5. Regarding lesions in cerebral toxoplasmosis:

(a) They are seen in patients with acquired immunodeficiency syndrome.

(b) They usually present as multiple hypodensities, with ring-like or nodular enhancement on CT.

(c) They are commonly located at the basal ganglia and corticomedullary junction.

(d) They can involve the corpus callosum and mimic butterfly glioma.

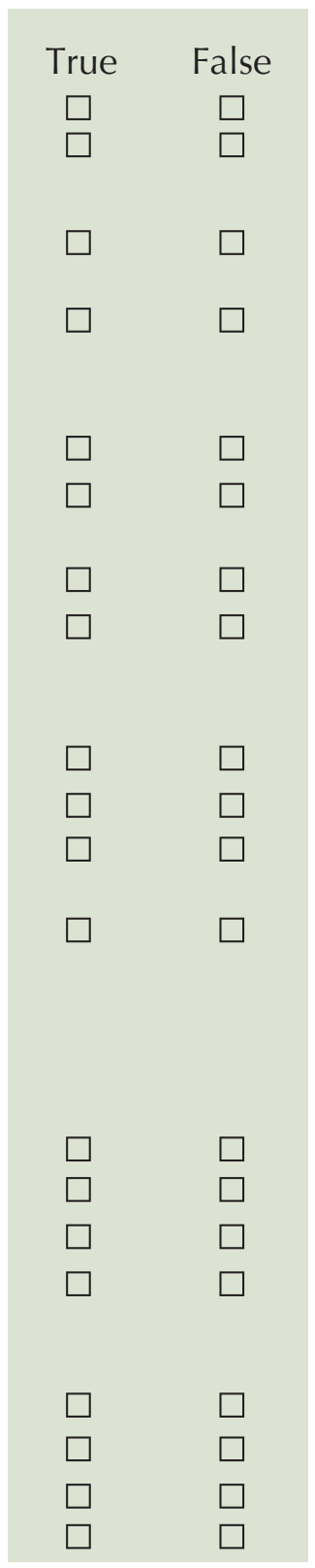

\footnotetext{
Doctor's particulars:

Name in full

MCR number

Specialty:

Email address

\section{SUBMISSION INSTRUCTIONS:}

(1) Visit the SMJ website: http://www.smj.org.sg/current-issue and select the appropriate set of questions. (2) Provide your name, email address and MCR number. (3) Select your answers and click "Submit".

\section{RESULTS:}

(1) Answers will be published online in the SMJ May 2017 issue. (2) The MCR numbers of successful candidates will be posted online at the SMJ website by 2 May 2017 . (3) Passing mark is $60 \%$. No mark will be deducted for incorrect answers. (4) The SMJ editorial office will submit the list of successful candidates to the Singapore Medical Council. (5) One CME point is awarded for successful candidates.

Deadline for submission: (March 2017 SMJ 3B CME programme): 12 noon, 25 April 2017.
} 